Relations industrielles

Industrial Relations

\title{
La sécurité syndicale et l'employeur
}

\section{Arthur Drolet}

Volume 2, numéro 8, avril 1947

URI : https://id.erudit.org/iderudit/1023876ar

DOI : https://doi.org/10.7202/1023876ar

Aller au sommaire du numéro

Éditeur(s)

Département des relations industrielles de l'Université Laval

ISSN

0034-379X (imprimé)

1703-8138 (numérique)

Découvrir la revue

Citer cet article

Drolet, A. (1947). La sécurité syndicale et l'employeur. Relations industrielles / Industrial Relations, 2(8), 6-8. https://doi.org/10.7202/1023876ar

Tous droits réservés @ Département des relations industrielles de l’Université Laval, 1947
Ce document est protégé par la loi sur le droit d'auteur. L’utilisation des services d'Érudit (y compris la reproduction) est assujettie à sa politique d'utilisation que vous pouvez consulter en ligne.

https://apropos.erudit.org/fr/usagers/politique-dutilisation/ 
rieur, seule la force peut clore la discussion. Et cette solution demeurera toujours éphémère, puisqu'elle ne se maintiendra plus lorsque le plus fort perdra ses positions.

Il est donc nécessaire, pour solutionner la question par la raison, de tenir compte des exigences collectives et de la hiérarchie entre les différents biens. Or le bien poursuivi par l'ouvrier qui ne veut point donner son adhésion au syndicat qui représente tous les travailleurs (souvent il possède une accréditation qui lui vient des organismes administratifs publics) et qui négocie au nom de tous une convention collective est sans contredit un bien particulier. De même, en est-il, pour l'employeur qui se refuse à ajouter cette condition supplémentaire devant être remplie soit par tous les travailleurs ou bien par quelques uns qui sont à son emploi. Et prenons la peine de remarquer qu'il ne s'agit que d'exceptions, car la majorité des travailleurs quand ce n'est pas davantage sont déjà membres du syndicat. Par ailleurs, le but poursuivi par le syndicat qui demande ces clauses d'affiliation syndicale est d'un ordre différent et supérieur. En effet, par ce moyen, le syndicat veut obtenir sa sécurité afin de travailler plus efficacement à l'intérêt de l'ensemble des travailleurs de telle entreprise déterminée.

Il convient cependant d'ajouter une remarque que nous considérons comme très importante. Nous croyons que le bien commun requiert que l'on ne se lie pas indéfiniment à une association quelconque: ce serait un véritable esclavage. Aussi est-il nécessaire qu'à certaines périodes données, il soit possible pour les travailleurs, non pas de se tenir à l'écart de tout groupement syndical, mais de choisir, s'ils le veulent, de donner leur adhésion à un autre groupement qui soit susceptible de promouvoir plus efficacement les intérêts de tout le groupe dans un établissement. Chez nous, dans la province de Québec, le cas ne se présente pas, puisque les conventions sont renouvelables chaque année et que la loi prévoit une période de temps bien déterminée au cours de laquelle les travailleurs peuvent changer leur adhésion syndicale.

Cependant, cette initiative d'insérer dans les conventions collectives des clauses qui obligent les travailleurs soit à adhérer au syndicat ou à le soutenir financièrement, quoiqu'elle puisse être prise par l'autorité publique, par l'employeur, par une confédération ou une fédération de syndicats ou enfin par un syndicat local, n'est généralement admissible que pour ce dèrnier, parce que seul, il a le droit de se considérer comme le représentant de l'ensemble des travailleurs dans une entreprise. Pour en arriver là, il lui est loisible d'utiliser tous les moyens honnêtes qu'il a à sa disposition, même les pressions économiques, puisque nous vivons dans un régime qui admet la concurrence entre les différents groupes et individus. Toutefois, si celui-ci a le droit de prendre les moyens de promouvoir sa sécurité, ce droit n'est pas non plus un droit absolu, inconditionné. Nous avons trop insisté au début de ce travail sur les limitations naturelles qui s'imposent à l'exercice de tout droit pour faire une exception en faveur du syndicat. Lui aussi, il s'intègre dans l'ensemble des institutions, il est soumis aux lois et la fin qu'il poursuit ne peut aller à l'encontre du bien commun. Le principe qui doit nous guider est le suivant: un syndicat ne doit jamais perdre de vue la raison même de son existence qui en même temps qu'elle se trouve à être le fondement de ses droits détermine aussi la limite de leur exercice. Voici, en résumé, les conditions qui doivent être remplies:

a) Le syndicat doit toujours respecter la liberté de conscience de ses membres et sa constitution doit permettre aux travailleurs de faire valoir leur point de vue d'après les usages communément admis dans les organisations démocratiques.

b) Le syndicat doit être réellement représentatif, poursuivre le bien de l'ensemble des travailleurs et n'être pas au service de certains individus ou d'une coterie quelconque.

c) Le syndicat ne peut en aucune façon profiter de cette force que lui donne l'adhésion et le soutien de tous les travailleurs soit pour des fins extra-syndicales ou pour propager des idées subversives.

d) Le syndicat ne peut utiliser cette mesure par malice ou par vengeance contre des individus ou contre un syndicat rival qui aurait la capacité d'assurer aux travailleurs les mêmes avantages soit dans l'entreprise ou dans la profession.

En somme, l'exercice de ce droit du syndicat à promouvoir sa sécurité par ces moyens doit être réservé à des groupements représentatifs, responsables, honnêtes, démocratiques, qui dans leur action tiennent compte des principes de la justice et subordonnent le tout au bien commun général

Avant de terminer, je voudrais souligner le fait que tout le long de ce travail, je n'ai considéré le problème que sous son aspect sociologique et moral, c'est-à-dire d'après le droit naturel. Voilà pourquoi la seule conclusion qui puisse se dégager, c'est que la sécurité syndicale établie par des clauses contractuelles dans les conventions collectives est légitime. Personne n'a donc le droit de se prévaloir uniquement de la liberté pour la condamner, pas plus le législateur quand il fait ses lois que l'employeur quand il négocie avec un syndicat. Si l'on veut s'y opposer raisonnablement, que l'on apporte d'autres motifs. Il est excessivement important de noter qu'en pratique, cependant, il faudra tenir compte de d'autres considérations. Trop souvent, on constate que ceux qui s'opposent à ces clauses de sécurité syndicale ont dit leur dernier mot quand ils ont parlé de la liberté et taxé la sécurité syndicale d'antidémocratique. Et, par ailleurs, ceux qui les préconisent après avoir tenté de prouver que la sécurité syndicale ne va pas à l'encontre de la liberté, qu'elle est aussi démocratique que toutes les autres mesures reconnues nécessaires en notre civilisation, en concluent immédiatement qu'il est opportun de l'établir. Dans l'un et l'autre cas, l'attitude pratique dépasse de beaucoup l'argumentation. Il nous semble quil soit nécessaire, pour saisir la question dans toute son ampleur d'envisager le problème sous d'autres aspects qui ne peuvent être dédaignés. Il faut tenir compte de la législation qui nous gouverne, des avantages ou des désavantages économiques qui s'en suivent pour le syndicat, pour l'entreprise, et pour le bien commun, car une chose, pour être bonne, doit l'être sous tous ses rapports.

Gérard DION

\section{LA SÉCURITÉ SYNDICALE ET L'EMPLOYEUR (Suite de la page 8)}

des cadres professionnels; et non l'esprit de lutte des classes; la conception matérialiste de la société ; la neutralité absolue à l'égard des principes religieux qui doivent exercer une influence sur la vie économico-sociale; autant de facteurs qui opposent les classes sociales et mènent vers le cataclysme où l'ordre de choses actuel sombrera pour faire place au collectivisme sinon au communisme.

Mes associés dans la production doivent être dirigés par des chefs d'une bonne formation sociale, bien éclairés sur les problèmes de l'heure; conscients de leurs responsabilités, calme et réfléchis dans l'étude et la défense des droits des travailleurs; pondérés lorsqu'il s'agit de promouvoir les intérêts de la classe ouvrière; prompts à l'action lorsqu'il leur faut prendre des mesures urgentes de discipline à l'égard de leurs membres : suffisamment doués pour comprendre les problèmes d'une industrie, d'une entreprise ; capables de présenter ces problèmes d'une manière objective à leurs syndiqués et les forcer à l'action constructive pour une collaboration efficace et soutenue.

Pour réaliser une telle action syndicale, il faut nécessairement que les chefs syndiqués possèdent une connaissance exacte des milieux sociaux dans lesquels ils évoluent. Il faut même davantage. Ils doivent vivre dans ces milieux afin de mieux saisir les éléments qui les composent: culture, influence religieuse, traditions familiales et sociales, conditions économiques, aspirations nationales, société politique, pouvoirs judiciaires, administratifs, législatifs, législations sociales. A moins d'être des nôtres, je ne vois pas comment des chefs de syndicats puissent comprendre tous ces facteurs, puissent sentir les diverses réactions des classes sociales, qui exercent une influence décisive dans l'action syndicale. 
Pour le progrès économique de l'entreprise et de la profession, l'employeur est en droit d'exiger un programme bien défini et pratique d'orientation professionnelle et d'apprentissage, de perfectionnement moral, social et professionnel de la main-d'oeuvre classifiée.

L'employeur réclame aussi une collaboration très étroite pour faire disparaitre l'absentéisme, pour faire comprendre à certains travailleurs leurs devoirs professionnels et sociaux d'accorder à l'entreprise, qui leur assure leur subsistance, un rendement satisfaisant. Collaboration aussi pour développer l'esprit d'équipe, d'entreaide, de coopération avec les surintendants et les contremaitres qui doivent recevoir une formation particulière à leurs fonctions, l'un des devoirs de l'employeur.

\section{Procédures patronales pour accorder la sécurité syndicale.}

On procèdera graduellement en accordant ces clauses de sécurité syndicale pour bien faire comprendre aux syndiqués qu'ils ne doivent pas compter uniquement sur l'employeur pour développer leur syndicat. Il faut que les employés s'occupent de leurs affaires, qu'ils consacrent tous leurs talents et leurs énergies à leur groupement syndical. Voici quelques suggestions:

A un syndicat qui vient de se fonder, on pourra accorder l'atelier préférentiel et peut-être le maintien d'affiliation, clause simple ou clause avec droit de retrait.

$\mathrm{Au}$ premier renouvellement, on pourra remplacer par une clause d'atelier syndical imparfait si le syndicat procède, avec succès, à l'éducation professionnelle et sociale de ses membres; s'il y a déjà à son crédit certaines initiatives pour le bénéfice des syndiqués par la mise en opération de certains services aux membres.

Subséquemment, l'atelier syndical parfait pourra être inséré dans la convention collective si l'influence du syndicat grandit et se fait sentir sans chocs, et qu'un certain pourcentage des non-syndiqués lors de la signature de la première convention ont adhéré depuis.

Enfin, l'atelier fermé, en temps opportun, lorsque le syndicat aura démontré que l'employeur peut compter sur lui pour lui procurer une main-d'oeuvre experte, disciplinée; lorsque le syndicat aura démontré qu'il a à coeur les intérêts professionnels, moraux et sociaux de ses membres par le développement des services d'orientation et de perfectionnement professionnel, de caisses de bien-être, de secours de toutes sortes.

Formule idéaliste me direz-vous ? L'organisation ouvrière, la meilleure qui existe présentement, n'est pas encore rendue à un tel degré de supériorité, par conséquent encore loin de cet idéal... L'employeur ne choisit pas l'organisation ouvrière qui négocie avec lui, il lui est même interdit d'en former ou de s'immiscer dans les organisations ouvrières. Voici qu'elle est ma réponse à ces deux objections.

L'histoire du mouvement ouvrier révèle les luttes incessantes livrées dans tous les pays industrialisés pour obtenir le droit de 's'associer, la négociation de la convention collective, la conciliation et l'arbitrage, la reconnaissance syndicale. Tour à tour ostracisés, considérés comme conspirateurs, les syndiqués ont dû engager des batailles légales et les gagner avec les maigres contributions des ouvriers mal payés. Aussi le syndicalisme sain, qui s'inspire de principes chrétiens, a dû d'abord déployer beaucoup d'efforts pour former et maintenir dans ses rangs des syndiqués trop enclins à porter leurs sympa- thies à des organisations ouvrières qui employaient la surenchère dans la négociation et parfois la démagogie pour en imposer par le nombre.

Quant à la deuxième objection, la-position respective du patronat et du travail changera d'une façon notable lorsque tous les deux seront organisés selon les mêmes principes et s'orienteront vers le même but. Le syndicalisme chrétien de notre province a fait naître, chez nous, un syndicalisme patronal identique. Des essais sont actuellement et heureusement tentés dans les trois genres d'activité économique de notre province: l'industrie, le commerce, les services.

Devant de telles associations patronales, le mouvement ouvrier devra présenter des garanties morales et professionnelles, être plus objectif, se bonifier même s'il y a lieu. De plus, un tel syndicalisme patronal collaborera davantage à la législation sociale et ouvrière.

\section{Conclusion.}

Un américain nous dit ce qu'il pense du problème des relations du Capital et du Travail. "Certes, nos relations du travail ne sont pas parfaites - et ne le seront jamais ! Mais si nous voulons bien cesser d'entraver le moyen de contact par excellence avec nos employés, plusieurs choses s'avéreront possibles, qui autrement ne pourront jamais être menées à bien :

1.-Nous pouvons traiter dans une atmosphère de négociation plus sociale et plus économique que pourrait le faire une lutte psychologique;

2.-Plus il entrera d'ouvriers américains dans le syndicat, plus ils seront assurés d'en déterminer la politique en élisant des chefs conformes aux idées américaines de démocratie et de loyauté ;

3.-Nous pouvons compter sur l'appui de l'ouvrier syndiqué américain pour corriger, par voie de législation ou autrement, la puissance effrénée et souvent destructive qu'exerce actuellement ce nouveau titan;

4.-Nous devons, pour notre survivance même sans parler de notre existence continue comme grande nation industrielle - nous devons restaurer nos relations du travail sur la base de l'unité d'intérèt et de la communauté de buts à atteindre. Nous devons exiger la démocratie dans l'industrie d'abord, si nous voulons qu'elle fleurisse dans notre société. Et plus même, nous devons ex'ger cette démocratie dans l'industrie américaine, si nous désirons qu'elle survive à la crise actuelle.

Si le syndicalisme doit être le moyen de contact par lequel nos employés exigent d'être représentés aux conseils de l'industrie, démontrons donc par notre largeur de vue, notre sincérité, et notre confiance en l'honnêteté et la noblesse de sentiments d'êtres humains, qui, s'ils sont des ouvriers lorsqu'ils travaillent à l'usine, sont aussi, et en tout temps, nos voisins, nos associés, nos concitoyens, prouvons donc, dis-je, que le syndicalisme est un organisme à bénéfices mutuels. ${ }^{3}$

Voilà pour les américains. Transposons ces idées dans notre milieu en y ajoutant les principes sociaux que nous devons appliquer et tous ensemble nous pourrons édifier un ordre social chrétien.

\section{Arthur Drolet}

(3) «abor Union are here to stay 》, par Arthur H. Young, California Institute of Technology. Extrait de \&The Closed Shop », The Reference Shelf, vol. 15, no 7, pp. 166-67. 


\section{LA SÉCURITÉ SYNDICALE ET L'EMPLOYEUR}

Pour mettre en valeur la puissance du nombre, la cohésion du Travail a été faite en vue de résoudre collectivement les problèmes que pose le fait de la production et de la distribution des richesses. Je laisse de côté beaucoup de thèses pour ou contre les groupements ouvriers, beaucoup d'objections de part et d'autre. Je m'arrête surtout à l'idée de groupements professionnels « réunissant les membres d'une même profession pour l'étude et la défense de leurs intérêts professionnels, et pour la règlementation de la profession en vue du bien de ses membres et du bien commun. »

«Ces groupements donnent à notre époque une physionomie nouvelle. Leur influence est grande sur la concurrence, qu'ils tempèrent, et sur l'organisation du travail. Bien conduits et animés d'un esprit de modération et d'équité, les syndicats peuvent améliorer la situation générale et contribuer à la pacification sociale. 》

"Que si, au contraire, les ouvriers coalisés abusent de leur puissance pour s'assurer des avantages immodérés ou pour réduire outre mesure la durée et l'intensité du travail, pour fomenter l'indiscipline dans les usines et pour limiter exagérément la production, le résultat de cette tactique sera désastreux pour la prospérité générale èt pour la classe ouvrière particulièrement. $\rrbracket^{1}$

\section{Rôle des groupements professionnels.}

Placés entre la famille et l'Etat, le groupement professionnel peut et doit contribuer à discipliner et à coordonner les forces du Travail et les faire collaborer efficacement à la production et au progrès de l'entreprise.

Pour accomplir ce rôle de collaborateurs immédiats, l'association ouvrière réclame la sécurité syndicale, c'està-dire «l'assurance de pouvoir continuer d'exister, de se perfectionner et de remplir efficacement son rôle de façon permanente. $»^{2}$

Le fait de la convention collective implique déjà la reconnaissance de l'association ouvrière, comme mandataire et fondée de pouvoir pour et au nom des travailleurs. Ces dirigeants ont étudié les besoins de ces travailleurs, l'importance de leur travail à l'usine, la compétence requise, les dangers dans tels secteurs de l'industrie ou l'effort physique requis pour certains travaux. Ils ont aussi évalué le salaire équitable qui devrait être versé pour telles ou telles catégories de travailleurs. Ils ont prié l'employeur de limiter le temps durant lequel les travailleurs seront requis à l'usine. De concert avec ces personnes accréditées, l'employeur s'est entendu et a signé un accord. L'employeur s'est associé avec un organisme auquel la majorité des travailleurs a confié ses intérêts. En fait, l'employeur reconnait que cet organisme possède, dans le milieu ouvrier et professionnel, une autorité, une

(1) Fallon, Principes d'économie sociale.

(2) Gérard Dion, Sécurité syndicale et convention collective, cahiers de la Faculté des sciences sociales, vol. IV, no 2, page 7 . influence. De plus, durant les mois qui ont suivi la signature de l'accord, l'employeur a constaté que l'assocration ouvrière a une influence sur ses membres, que ces derniers ont donné certaines preuves de discipline à suivre les directives des chefs. Quelques difficultés dans l'interprétation et l'application des clauses du contrat ont été réglées avec un esprit de collaboration et une réelle préoccupation de promouvoir la bonne entente.

L'employeur se demande alors s'il n'y a pas lieu de faire béneticier davantage son entreprise de cette intluence par des mesures propres à assurer le développement et la permanence de cet organisme de collaboration.

\section{La sécurité syndicale.}

Mais résumons d'abord les objections les plus courantes des employeurs à ces clauses de sécurité syndicale:

L'autorité patronale est partiellement sinon totalement méconnue.

Les employés ne sont plus libres d'appartenir à l'union de leur choix ou de demeurer isolés de tout groupement.

Les employés doivent subir une autorité arbitraire dans l'embauchage et le renvoi.

Les employeurs sont forcés de refuser du travail à des ouvriers compétents parce que ces derniers veulent conserver leur liberté d'appartenir ou non à une union.

Les employés sont contrôlés par un petit groupe d'officiers et d'agents d'affaire qui se paient de gros salaires aux dépens des ouvriers.

Les employés sont obligés de faire la grève, selon les directives des chefs de l'union.

Les employeurs sont obligés de signer des conditions de travail et de salaire exagérées, sous la menace de la grève.

Les unions empêchent les patrons de s'approcher des ouvriers, de leur accorder des avantages personnels pour aider à faire vivre leur famille.

Ainsi donc, lorsque l'employeur s'engage avec une association ouvrière à une clause d'atelier fermé, il accorde à celle-ci un tel pouvoir que dans l'éventualité d'une rupture des relatior,s, les plus graves conséquences peuvent en résulter. Pourtant, des expériences ont été faites sans ces ennuis et qui ont procuré même des avantages aux industries qui ont négocié de telles clauses de sécurité syndicale. Mais avant de conclure de telles ententes, l'employeur a l'impérieux devoir d'étudier le problème sous tous ces aspects et d'essayer d'en prévoir les avantages et aussi les conséquences. Il a le droit de connaitre aussi parfaitement que possible l'organisme avec lequel il aura à transiger. Il a aussi le droit d'exiger des garanties. Ce n'est pas tant pour lui-même, pour ses héritiers ou pour ceux qui lui ont donné un mandat, que l'employeur s'engage. Il engage l'entreprise, l'institution, qui doit continuer à servir la collectivité.

\section{Responsabilité du syndicat dans \\ la profession et l'entreprise.}

L'association ouvrière doit avoir de solides principes sociaux qui guident son action vers la collaboration dans (Suite à la page 6) 